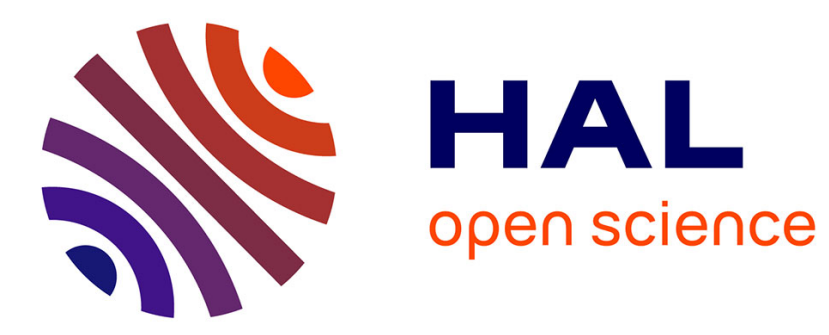

\title{
Growth by rectified diffusion of strongly acoustically-forced gas bubbles in nearly saturated liquids
}

Olivier Louisnard, Francisco Gomez

\section{- To cite this version:}

Olivier Louisnard, Francisco Gomez. Growth by rectified diffusion of strongly acoustically-forced gas bubbles in nearly saturated liquids. Physical Review E: Statistical, Nonlinear, and Soft Matter Physics, 2003, vol. 67 (n 3), pp. 036610. 10.1103/PhysRevE.67.036610 . hal-01343141

\author{
HAL Id: hal-01343141 \\ https://hal.science/hal-01343141
}

Submitted on 7 Jul 2016

HAL is a multi-disciplinary open access archive for the deposit and dissemination of scientific research documents, whether they are published or not. The documents may come from teaching and research institutions in France or abroad, or from public or private research centers.
L'archive ouverte pluridisciplinaire HAL, est destinée au dépôt et à la diffusion de documents scientifiques de niveau recherche, publiés ou non, émanant des établissements d'enseignement et de recherche français ou étrangers, des laboratoires publics ou privés. 


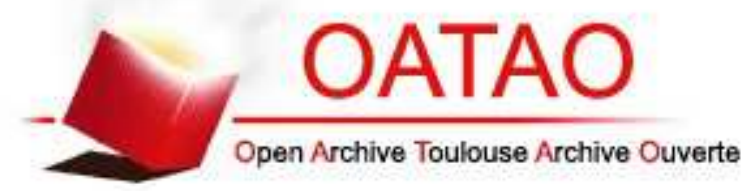

\section{Open Archive TOULOUSE Archive Ouverte (OATAO)}

OATAO is an open access repository that collects the work of Toulouse researchers and makes it freely available over the web where possible.

This is an author-deposited version published in: http://oatao.univ-toulouse.fr/ Eprints ID : 15929

To link to this article : DOI:10.1103/PhysRevE.67.036610

URL : http://dx.doi.org/10.1103/PhysRevE.67.036610

\section{To cite this version :}

Louisnard, Olivier and Gomez, Francisco Growth by rectified diffusion of strongly acoustically-forced gas bubbles in nearly saturated liquids. (2003) Physical Review E, vol. 67 (n³). pp. 036610. ISSN 1539-3755

Any correspondence concerning this service should be sent to the repository administrator: staff-oatao@ listes-diff.inp-toulouse.fr 


\title{
Growth by rectified diffusion of strongly acoustically-forced gas bubbles in nearly saturated liquids.
}

\author{
Olivier Louisnard* \\ Laboratoire de Génie des Procédés des Solides Divisés, UMR CNRS 2392, \\ Ecole des Mines d'Albi, 81013 Albi Cedex 09, France \\ Francisco Gomez ${ }^{\dagger}$ \\ Laboratorio de Ultrasonidos, \\ Universidad de Santiago de Chile, \\ Casilia 30\%, Santiago 2, Chile
}

\begin{abstract}
The growth or dissolution of small gas bubbles $\left(R_{0}<15 \mu \mathrm{m}\right)$ by rectified diffusion in nearly saturated liquids, subject to low frequencies $(20 \mathrm{kHz}<f<100 \mathrm{kHz}$ ), high driving acoustic fields (1 bar $<p<5$ bar) is investigated theoretically. It is shown that, in such conditions, the rectified diffusion threshold radius merges with the Blake threshold radius, which means that a growing bubble is also an inertially-oscillating bubble. On the assumption that such a bubble keeps its integrity up to the shape instability threshold predicted by single-bubble theory, a numerical estimation, and a fully analytical approximation of its growth-rate are derived. From one hand, the merging of the two thresholds raises the problem of the construction and self-sustainment of acoustic cavitation fields. From the other hand, the lifetime of the growing inertial bubbles calculated within the present theory is found to be much shorter than the time necessary to rectify argon. This allows an alternative interpretation of the absence of single-bubble sonoluminescence (SBSL) emission in multi-bubble fields, without resorting to the conventional picture of shape instabilities caused by the presence of other bubbles.
\end{abstract}

PACS numbers: 47.55.Bx, 43.35.+d, 78.60.Mq

\section{INTRODUCTION}

The characteristics of the radial oscillations of a gas bubble in a sound field are mainly governed by three parameters: the pressure amplitude of the acoustic field, its frequency, and the bubble ambient radius, related to the mass of gas contained in the bubble.

Two distinct dynamic bubble behaviors may be encountered: stable cavitation depicts possibly nonlinear, smooth oscillations, whereas inertial cavitation is characterized by an explosive growth of the bubble during the wave rarefaction phase, followed by a violent collapse. For sufficiently low frequency and small bubbles (more precisely for bubbles much smaller than the resonant radius), a quasi-static argument may be used to derive the frontier between the two behaviors in the parameter space, the so-called Blake threshold $[1,2]$. The relevance of this threshold to distinguish two different dynamic behaviors has been proved numerically $[3,4]$.

The oscillatory convective-diffusive gas transfer between the bubble and the liquid may reverse in some cases the natural tendency of a gas bubble to dissolve under surface tension. This process known as rectified diffusion originates from the non-zero average gas transfer between the bubble and the liquid over the expansion and contraction phases of the bubbles $[1,5-8]$. As surface

\footnotetext{
*Electronic address: louisnar@enstimac.fr

$\dagger$ Electronic address: fgomezf2002@yahoo.es
}

tension still contributes to the bubble dissolution, bubble growth occurs when the acoustic pressure exceeds some threshold value. This rectified diffusion threshold (referred hereafter as RD threshold) also cuts the parameter space in two parts: dissolving and growing bubbles.

Each threshold is generally defined as a critical value of the acoustic pressure for a fixed bubble radius. However, since the latter is not a free parameter in a cavitation experiment, it is more convenient to define a threshold radius for a fixed acoustic pressure. Such thresholds may serve to describe the history of a bubble in the sound field [9], referred to as the cavitation cycle (see Ref. 10 for an extensive discussion). In nearly saturated liquids and sufficiently high pressure drives, a commonly accepted scenario is that a small, smoothly-oscillating bubble, grows by rectified diffusion up to the Blake threshold, where it starts oscillating inertially. In fact, as will be shown in the first part of this paper, for near saturation conditions and low frequencies, the RD and Blake thresholds merge for decreasing bubble radii, or conversely for increasing driving pressures. This means that, in order to grow, a bubble should be driven above the Blake threshold, and therefore oscillate inertially.

The next question arising is the lifetime of such an inertial-growing bubble, and this raises the issue of the shape stability of inertial bubbles. Up to the first single bubble sonoluminescence (SBSL) experiments [11], it was generally accepted that an inertial bubble could only survive a few collapses before bursting by shape instabilities. However, the lifetime of a SBSL bubble is in itself an experimental proof of the existence of shape-stable inertial 
bubbles. This has been confirmed by recent theoretical work dedicated to shape instabilities [12-15] based on earlier studies [16, 17], and confronted to experimental data in SBSL experiments $[18,19]$. For example, when submitted to an acoustic pressure of 1.5 bar, and a frequency of $32.8 \mathrm{kHz}[15,19]$, any single bubble smaller than about $5 \mu \mathrm{m}$ should be shape stable. Since the Blake threshold is about $1 \mu \mathrm{m}$ in this case, a relatively large range of sizes for shape-stable inertial bubbles is allowed.

There subsists some doubt on the existence of inertial, shape-stable bubbles, in the context of multi-bubble fields, for which the single bubble shape-stability theory may not be relevant, owing to perturbations caused by neighboring bubbles [20]. However, several experimental results in $20 \mathrm{kHz}$ cavitation fields report distributions of bubble ambient radii ranging from $1 \mu \mathrm{m}$ to $10 \mu \mathrm{m}$ [21, 22], which is in rough agreement with the above estimation. Therefore, as a general hypothesis, we will assume that the instability thresholds calculated in the SBSL context keep their validity in multi-bubble fields. Although not mentioned explicitly, the hypothesis of the survival of inertial bubbles to many collapses has been used by other investigators dealing with multi-bubble fields [21, 23].

In a nearly saturated liquid, the lifetime of the inertial growing bubbles would therefore correspond to their growth-time by rectified diffusion between the common RD-Blake threshold ( $1 \mu \mathrm{m}$ in the above example) and the shape instability threshold ( $5 \mu \mathrm{m}$ in the above example). The estimation of this lifetime requires the knowledge of the growth-rate of an inertial bubble and the second part of this paper is dedicated to this topic. By using the results of Fyrillas and Szeri [8], the growth-rate will be calculated numerically by scanning the parameter space and a fully explicit analytical formula will also be proposed for practical applications.

To illustrate the theory on a practical example, we will consider in this paper air-bubbles in nearly saturated water. The following region of the parameter space will be studied: low frequencies (say lower than $100 \mathrm{kHz}$ ), and high driving pressures (from 1 to 5 bar), which are commonly used conditions in sonochemistry and cavitation experiments. In view of the order of magnitude of the shape-instability threshold and of the experimentally measured size distributions [21, 22], our calculations will be performed for bubble radii ranging between the Blake threshold and $15 \mu \mathrm{m}$.

\section{THEORY}

\section{A. Bubble model}

The radial oscillations of a gas bubble in a liquid may be described by the Rayleigh-Plesset (RP) equation:
$R \ddot{R}+\frac{3}{2} \dot{R}^{2}=\frac{1}{\rho}\left(p_{g}+\frac{R}{c_{l}} \frac{d p_{g}}{d t}-4 \mu \frac{\dot{R}}{R}-\frac{2 \sigma}{R}-p_{\text {ext }}(t)\right)$.

In this equation $p_{\text {ext }}(t)=p_{0}(1-p \cos \omega t)$ is the oscillating sound pressure in the liquid, with $\omega$ angular frequency, $p$ dimensionless acoustic pressure and $p_{0}$ hydrostatic pressure; $\rho, \mu$ and $c_{l}$ are the density, viscosity and sound speed of the liquid; $\sigma$ is the surface tension. The gas pressure $p_{g}(t)$ may be reasonably assumed uniform [24] and to obey a van der Waals state equation:

$$
p_{g}=\left(p_{0}+\frac{2 \sigma}{R_{0}}\right)\left(\frac{R_{0}^{3}-h^{3}}{R^{3}-h^{3}}\right)^{\kappa},
$$

where $R_{0}$ is the ambient radius of the bubble and $h$ the van der Waals hard-core radius. We will also assume that the bubble follows isothermal evolutions $(\kappa=1)$. This approximation may be considered as drastic in view of more complete theories $[25,26]$, but has been used successfully by Hilgenfeldt and co-authors [4, 12] to derive the phase diagram of SBSL. In what follows we will make use of the dimensionless time and dimensionless bubble radius:

$$
x=\omega t, \quad R^{*}=R / R_{0},
$$

and we define the dimensionless Laplace tension:

$$
\alpha_{S}=\frac{2 \sigma}{p_{0} R_{0}}
$$

\section{B. Blake threshold}

The expression of the the Blake threshold, separating smooth oscillating bubbles from inertial ones, may be obtained by calculating the minimal pressure $p_{\text {ext }}$ that the bubble can withstand without fluid motion $[1,2]$. Expressing the result as a critical dimensionless acoustic pressure yields:

$$
p_{B}=1+\left(\frac{4}{27} \frac{\alpha_{S}^{3}}{1+\alpha_{S}}\right)^{1 / 2}
$$

Conversely, for a given acoustic pressure $p$, a Blake threshold radius can be obtained by solving equation (5), which is cubic for $\alpha_{S}$. The detailed calculation may be found in Ref. 4. Suffice here to note that the parameter $\alpha_{S}$ becomes $O(1)$ for $p_{B}$ as low as 1.2. This means that Laplace tension becomes important for a bubble near the Blake threshold radius, for any acoustic pressure greater than 1.2 bar. We will make use of this result in section III B. 


\section{Rectified diffusion}

The asymptotic time-averaged variation of the bubble gas-content can be calculated by use of the results of Fyrillas and Szeri [8]. In order to solve the convectiondiffusion PDE describing the movement of the dissolved gas, they split the problem into a smooth one, describing the rectified mass variation of the bubble, and an oscillatory one, describing the zero-average mass exchange occurring over one bubble oscillation. On the assumption that the time-scales of the two process are well separated, they obtained:

$$
\frac{d m_{g}}{d \tau}=4 \pi D R_{0} C_{0} \frac{C_{\infty}^{*}-\left\langle p_{g} / p_{0}\right\rangle_{4}}{I}
$$

where $\tau$ is the slow mass-variation time-scale $(\gg 2 \pi / \omega)$, $D$ is the gas-diffusivity in the liquid, $C_{0}$ the saturation concentration in the liquid separated from gas at pressure $p_{0}$ by a plane boundary, and $C_{\infty}^{*}=C_{\infty} / C_{0}$ with $C_{\infty}$ gas concentration in the liquid far from the bubble. The quantity $I$, whose evaluation will be detailed in section III C, denotes the spatial integral

$$
I=\int_{0}^{+\infty} \frac{d s}{\left\langle\left(3 s+R^{* 3}\right)^{4 / 3}\right\rangle_{1}} .
$$

The weighted averages \langle\rangle$_{i}$ occurring in the above expressions are defined by:

$$
\langle f\rangle_{i}=\frac{\int_{0}^{2 \pi} f(x) R^{i}(x) d x}{\int_{0}^{2 \pi} f(x) d x},
$$

and can be calculated from the solution $R^{*}(x)$ of the RP equation (1).

The fraction in expression (6) is the dimensionless nonlinear-averaged gas concentration gradient between an infinitely far point and the bubble wall.

The locus of diffusive equilibrium in the parameter space $\left(R_{0}, p\right)$ is defined by:

$$
C_{\infty}^{*}-\left\langle p_{g} / p_{0}\right\rangle_{4}=0 .
$$

For sufficient degassing, this equilibrium may turn to be stable. This is the case of a SBSL bubble, which adjusts its ambient radius to the driving level [12]. When it is unstable, it defines the rectified diffusion threshold: any bubble larger than this threshold will grow, and any bubble smaller will dissolve. A successful comparison of Eq. (9) with experimental data for saturated or nearsaturated conditions may be found in Ref. [8] for bubble radii down to $20 \mu \mathrm{m}$. Besides, in degassed conditions, the good agreement between the theoretical phase diagrams and SBSL data $[12,14]$ support the validity of Eq. (9) also for inertial oscillations.

It should be added that the rigorous approach of Fyrillas and Szeri [8] allows to obtain an uniformly valid expression of the growth-rate, even far from the threshold, contrary to earlier results $[5,6]$ whose derivation relied either on linear oscillations, or on near-threshold conditions. We refer the interested reader to Ref. 8 for an extensive discussion of this topic.

\section{RESULTS}

\section{A. Merging of RD and Blake threshold}

We consider through this paper air bubbles in ambient water $\left(p_{0}=1\right.$ bar, $\rho=1000 \mathrm{~kg} \cdot \mathrm{m}^{-3}, c=1481 \mathrm{~m} \cdot \mathrm{s}^{-1}$, $\mu=10^{-3}$ Pa.s, $\left.\sigma=0.073 \mathrm{~kg} . \mathrm{s}^{-2}, h=R_{0} / 8.76\right)$. The $\mathrm{RD}$ threshold was computed by tracking the locus of the points fulfilling condition $(9)$ in the $\left(R_{0}, p\right)$ plane. For each $\left(R_{0}, p\right)$ point, the average $\left\langle p_{g} / p_{0}\right\rangle_{4}$ was calculated from Eq. (2) using the steady-state numerical results of the RP equation.

Figure 1 shows the RD threshold, computed from the RP equation (thick solid line) for saturated water $\left(C_{\infty}^{*}=1\right)$ in a $26.5 \mathrm{kHz}$ acoustic field. Also shown is the threshold calculated from the linear theory of Crum and Hansen [7] (thick dot-dashed): it is readily seen that for bubbles of small ambient radii, the linear theory fails predicting the pressure threshold value, but for larger bubbles the exact threshold merges with the linear one, at least in the range of radii considered here. More surprising is the comparison of the RD threshold with the Blake threshold, also displayed in Fig. 1 (thick dashed line): it is seen that the two thresholds merges for acoustic pressure greater than, say 1.4 bar, for saturated water. Performing the same calculations for slightly degassed water $\left(C_{\infty}^{*}=0.8\right)$ yields the same conclusion, as attested by Fig. 1 (the numerical RD threshold is represented by a thin solid line and the linear one by thin dot-dashed line). The computation was also repeated for $50 \mathrm{kHz}$ and $100 \mathrm{kHz}$ frequencies, leading to the same conclusion (not shown).

This singular result can be interpreted more easily in view of the average bubble pressure $\left\langle p_{g} / p_{0}\right\rangle_{4}$ curves represented in Fig. 2: the equilibrium points defined by Eq. (9) for a given gas concentration $C_{\infty}^{*}$ are the intersection of these curves and the horizontal line $\left\langle p_{g} / p_{0}\right\rangle_{4}=C_{\infty}^{*}$. For degassed water $\left(C_{\infty}^{*} \ll 1\right.$, dot-dashed line), a stable equilibria may be obtained (right intersection point), a result largely mentioned in the SBSL literature [3, 4]. For $C_{\infty}^{*}=1$ (thin solid line), it can be seen that the intersection points almost merges with the Blake radius (represented by vertical dashed line for each driving pressure).

More physically, this behavior should be understood as an effect of surface tension which is important for bubbles of small radii $\left[\alpha_{S}=O(1)\right]$. Bubbles lower than the Blake threshold radius are prevented to expand, and stay in a quasi-linear oscillation state. In this case the average gas pressure $\left\langle p_{g} / p_{0}\right\rangle_{4}$ is dominated by surface tension and the bubble dissolves. Thus, the only way for a such a small bubble to gain some gas on average over an acoustic cycle 
is to oscillate inertially, which of course is possible if the acoustic forcing is sufficiently high.

Conversely, bubbles much larger than the Blake radius, so that surface tension plays a negligible role $\left(\alpha_{S} \ll 1\right)$, will be allowed to grow freely for moderate driving pressures, thus rendering the linear theory valid. This is why the exact rectified diffusion threshold merges with the linear one in the right part of Fig. 1.

\section{B. Analytical justification}

The merging between the RD and Blake threshold may be explained by the following analytic arguments. Let's first reformulate the threshold condition to make clear the role of surface tension:

$$
C_{\infty}^{*}-\left(1+\alpha_{S}\right)\left\langle p_{g} / p_{g_{0}}\right\rangle_{4}=0,
$$

where $p_{g_{0}}=p_{0}+2 \sigma / R_{0}$ is the ambient gas pressure in the bubble.

We first consider the case of smooth quasi-linear oscillations: in this case, the bubble radius $R(t)$ departs only slightly from its ambient radius $R_{0}$, and so does the bubble pressure. Therefore, $p_{g}(t) \simeq p_{g_{0}}$ and we may write $\left\langle p_{g} / p_{g_{0}}\right\rangle_{4}=1+\epsilon$, where $\epsilon$ is a small parameter. The threshold condition Eq. (10) reads in this case:

$$
C_{\infty}^{*}-\left(1+\alpha_{S}\right)(1+\epsilon)=0,
$$

which can only be fulfilled if $C_{\infty}^{*}=O(1)$ and $\alpha_{S}=O(\epsilon)$. This brings the conclusion that a weakly oscillating bubble can grow only in near-saturated water, and for small Laplace tension.

Now, in the case of an inertial bubble, the average gas pressure $\left\langle p_{g} / p_{g_{0}}\right\rangle_{4}$ may be shown to scale as [4, 27]:

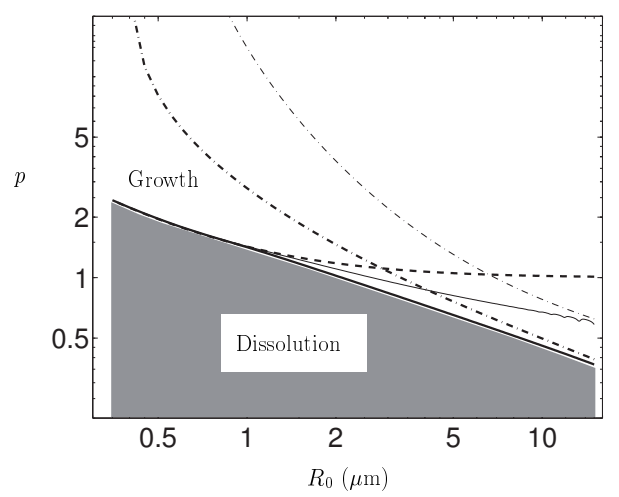

FIG. 1: Exact RD threshold for saturated water $\left(C_{\infty}^{*}=1\right)$ calculated from RP equation and Eq. (9) (thick solid line); Blake threshold from Eq. (5) (thick dashed line); linear theory RD threshold calculated from Ref. 7 (thick dot-dashed line). The thin lines represent respectively the exact (solid) and linear (dot-dashed) RD thresholds calculated for slightly degassed water $\left(C_{\infty}^{*}=0.8\right)$.

$$
\left\langle p_{g} / p_{g_{0}}\right\rangle_{4}=\xi\left(R_{0} / R_{\max }\right)^{3}
$$

where $R_{\max }$ is the maximum radius of the bubble over the acoustic cycle and $\xi=105 / 64$. Since $R_{\max }$ is several times greater than $R_{0}$ for inertial cavitation, $\left\langle p_{g} / p_{g_{0}}\right\rangle_{4}$ is therefore a small parameter $\epsilon$ so that the threshold condition now reads

$$
C_{\infty}^{*}-\left(1+\alpha_{S}\right) \epsilon=0
$$

and can only be fulfilled if $C_{\infty}^{*}=O(\epsilon)$ : this is the SBSL case and shows why degassing is necessary to get diffusive equilibrium (which turns to be stable in some cases). Conversely, if $C_{\infty}^{*}=O(1)$, the quantity $C_{\infty}^{*}-\left(1+\alpha_{S}\right) \epsilon$ is always positive, so that the inertial bubble grows.

From the above discussion, the diffusive behavior of a bubble near the Blake threshold for $C_{\infty}^{*}=O(1)$ may now be inferred: from section II B, $\alpha_{S}=O(1)$ for a bubble near the Blake threshold radius. Therefore, the above analysis tells us that i) a weakly oscillating bubble dissolves ii) an inertial bubble grows. The RD threshold is thus the limit between smooth and inertial oscillation, and this is precisely the definition of the Blake threshold.

The occurrence of crossing between the RD and Blake thresholds has been mentioned in the literature [28], but to our knowledge, the merging of the two curves for saturated water in the present parameter range has never been reported.

Having shown that a growing bubble in near saturation water should also be an inertial bubble, we now turn to calculate its growth-rate.

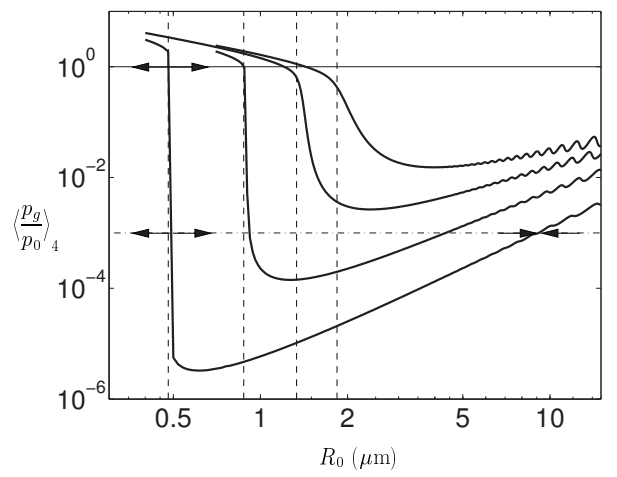

FIG. 2: Time-average pressure $\left\langle p_{g} / p_{0}\right\rangle_{4}$ vs. ambient radius for driving pressures $p=1.2,1.3,1.5$, and 2 from top to bottom. The horizontal lines represent gas concentrations in the liquid; thin solid: $C_{\infty}^{*}=1$; dot-dashed: $C_{\infty}^{*}=0.001$. The vertical dashed lines represent the Blake threshold for the different driving pressures: $p=1.2,1.3,1.5$, and 2 from right to left. The arrows on the lower curve $(\mathrm{p}=2)$ indicate bubble growth or dissolution regions. 


\section{Growth-rate}

\section{Approximation for integral I}

The calculation of the growth-rate involves the evaluation of the definite integral $I$. To our knowledge, only two references report results involving the computation of this quantity: Fyrillas and Szeri [8] reports growth-rates without indicating the method of calculation; Hilgenfeldt et al. [12] mention its slow convergence and propose an empirical formula involving adjustable parameters with few computational details. We present below both approximate analytical and numerical calculations of this integral for a large parameter set.

The derivation of the analytical approximation follows the spirit of Ref. 4: we propose a scaling law for integral $I$ in terms of the maximum radius attained by the bubble. The underlying idea of our derivation is made clear in Fig. (3) which displays, for a typical inertial cavitation bubble, the bubble radius, $R^{*}(x)$, and the bubble volume, $R^{* 3}(x)$, which is the quantity involved in expression (7). It is seen that the global pulse-like shape of the bubble volume is dominated by the expansion and collapse phases, and that the afterbounces are of minor importance. Thus, it may be expected that the time-average involved in expression (7) will be controlled mainly by $R_{\max }^{*}$, but also by the half-width $\Delta x$ of the "pulse". We therefore approximate the bubble volume by a parabola of maximum $R_{\max }^{*}{ }^{3}$ and half-width $\Delta x$, and introduce this approximate bubble dynamics in Eq. (7). The detailed calculation is shifted in appendix A and it is shown that integral $I$ writes approximately:

$$
I \simeq \frac{1}{3 R_{\max }^{*}} \frac{A}{\left(x_{m}-\alpha x_{+}\right)^{e}}
$$

where $R_{\max }^{*}=R_{\max } / R_{0}$ is the dimensionless maximum bubble radius, $x_{m}$ is the time of maximum expansion of the bubble, $x_{+}=\arccos (1 / p)$ is the zero-crossing of the acoustic pressure (see Fig. 3c), and $\alpha$ is a correcting factor to take into account the non parabolic shape of $R^{* 3}(x)$. Both parameters $A$ and $e$ originate from the power-law fit of an integral involving hypergeometric functions [see Eq. (A11)].

In order to obtain a full analytical expression for $I$, which can be evaluated in terms of the parameters $\left(R_{0}, p, \omega\right)$, we further make use of approximate expressions of $R_{\max }^{*}$ and $x_{m}$ obtained by Hilgenfeldt and coauthors [4]. The method used by these authors and the refined formulae we use in this paper are reported in appendix C. We simply recall below the general form of these approximate expressions:

$$
\begin{aligned}
R_{\max }^{*} & =\left[f(p)+\left(1-\frac{4}{9 \sqrt{3}} \frac{\alpha_{S}}{p-1}\right) g(p)\left(\frac{R_{\mathrm{res}}}{R_{0}}\right)^{2}\right]_{(15 \mathrm{a})}^{1 / 2} \\
x_{m} & =h(p),
\end{aligned}
$$

where $R_{\text {res }}=\left(3 p_{0} / \rho \omega^{2}\right)^{1 / 2}$ is the isothermal resonant radius, and $f, g$ and $h$ are given by Eqs. (C3a), (C3b) and (C6) respectively.

Besides, the numerical calculation of $I$ is obtained by scanning the parameter space and solving the RP differential equation up to a steady state. The bubble dynamic $R^{*}(x)$ thus obtained is then used to calculate integral (7), using a Gauss quadrature method described in appendix B.

It is shown in appendix A that the set of approximations (14)-(15) shows excellent agreement with the exact numerical value of integral $I$ (Figs 7 and 8).

\section{Approximation for the growth-rate}

With the above analytical formula for $I$, the growthrate itself may now be calculated easily. From Eq. (6), the slow time-variation of the bubble radius reads [7]:

$$
\frac{d R_{0}}{d \tau}=\frac{D R_{G} T C_{0}}{p_{0}} \frac{1}{R_{0}\left(1+2 / 3 \alpha_{S}\right)} \frac{C_{\infty}^{*}-\left\langle p_{g} / p_{0}\right\rangle_{4}}{I}
$$

Using Eq. (14) for integral $I$ and Eq. (12) to approximate the average gas pressure, we get

$$
\begin{aligned}
\frac{d R_{0}}{d \tau}= & \frac{3}{A} \frac{D R_{G} T C_{0}}{p_{0}}\left[C_{\infty}^{*}-\left(1+\alpha_{S}\right) \xi R_{\max }^{*}{ }^{-3}\right] \\
& \times \frac{R_{\max }^{*}}{R_{0}\left(1+2 / 3 \alpha_{S}\right)} F_{1}(p)
\end{aligned}
$$

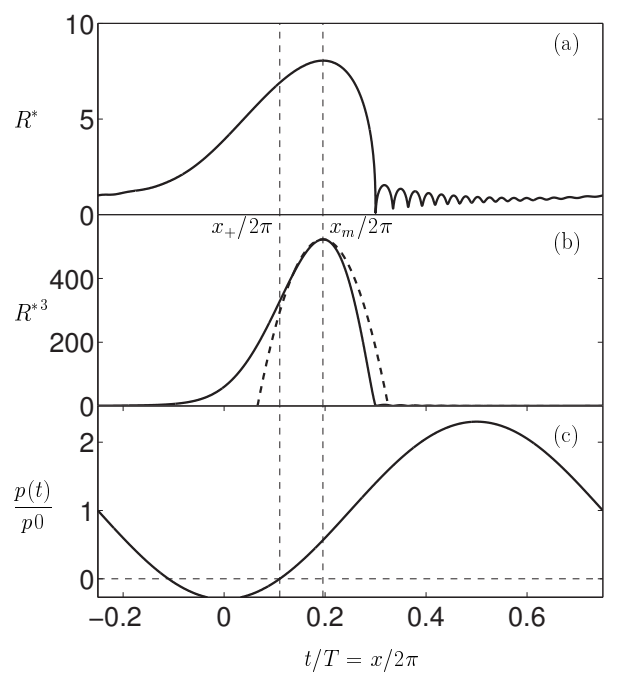

FIG. 3: (a) Bubble radius $R^{*}$; (b) Bubble volume $R^{* 3}$; (c) Dimensionless driving pressure $p_{\text {ext }}(x) / p_{0}=(1-p \cos x)$. The case considered is a $5 \mu \mathrm{m}$ air bubble in water and $p=1.3$. The times $x_{+}$and $x_{m}$ are respectively the instant of zero-crossing of the driving pressure and the time of maximum expansion of the bubble. The dashed curve in (b) is the parabolic approximation Eq. (A5) with $\Delta x$ defined by Eq. (A13). 
where $R_{\max }^{*}$ is calculated from Eq. (15a), and

$$
F_{1}(p)=[h(p)-\alpha \arccos (1 / p)]^{e} .
$$

Figure 4 displays a comparison between the exact numerical result, calculated by injecting the RP solution in Eq. (16) (thick solid line), and the approximation (17) (thin solid line). It is seen that our approximation yields very good results for acoustic pressures up to 5 bars and ambient radii up to $20 \mu \mathrm{m}$, apart for the wiggles appearing on the $R_{0}=10 \mu \mathrm{m}$ and $R_{0}=20 \mu \mathrm{m}$ numerical curves. These wiggles find their origin in the mode-locking of the bubble afterbounces [4] and are not accounted for by approximation (15a) of $R_{\max }^{*}$.
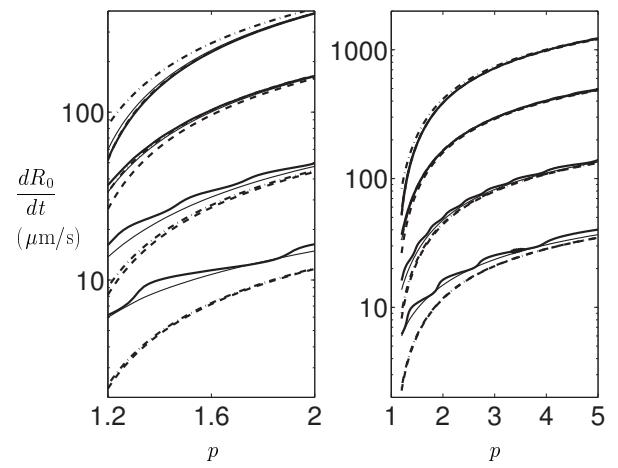

FIG. 4: Calculated value of the bubble growth-rate for different bubble sizes from exact result (16) (thick solid line); from (17) (thin solid line); from (19) (dashed line); from (21) (dot-dashed line). From top to bottom: $R_{0}=3,5,10$, and 20 $\mu \mathrm{m}$. The left figure is a magnification of the right one.

In the specific case of cavitation field experiments, where generally the liquid is gas saturated $\left.\left[C_{\infty}^{*}=O(1)\right)\right]$, the above formula may be further simplified: as mentioned above, for inertial cavitation, $R_{\max }^{*} \gg 1$ so that it may be neglected in the bracket of Eq. (17). It is interesting to note that this approximation should hold only far from the RD threshold, but as seen in the first section, the RD and Blake thresholds are the same. Since the average gas pressure curves near the threshold are very steep (see Fig. 2), any bubble driven just above this threshold may be considered far from it, in the sense that $\left\langle p_{g} / p_{0}\right\rangle_{4} \gg C_{\infty}^{*}$.

Moreover, for sufficiently low frequency, the Blake radius is much smaller than the resonant radius so that $R_{0} \ll R_{\text {res }}$ and $f(p)$ may be safely neglected in Eq. (15a). Replacing the obtained value for $R_{\max }$, we get the approximation:

$$
\frac{d R_{0}}{d \tau}=\frac{3}{A} \frac{D R_{G} T C_{\infty}}{p_{0}} \frac{\sqrt{\left(1-\frac{4}{9 \sqrt{3}} \frac{\alpha_{S}}{p-1}\right)}}{\left(1+2 / 3 \alpha_{S}\right)} \frac{R_{\mathrm{res}}}{R_{0}^{2}} F_{2}(p),
$$

where

$$
F_{2}(p)=\sqrt{g(p)}[h(p)-\alpha \arccos (1 / p)]^{e} .
$$

For sufficiently large $p$ and large $R_{0}$, the square-root may be further replaced by 1 , yielding the simpler expression:

$$
\frac{d R_{0}}{d \tau}=\frac{3}{A} \frac{D R_{G} T C_{\infty}}{p_{0}} \frac{1}{\left(1+2 / 3 \alpha_{S}\right)} \frac{R_{\mathrm{res}}}{R_{0}^{2}} F_{2}(p) .
$$

Figure 4 also shows the growth-rates given by Eqs. (19) (dashed line) and (21) (dot-dashed line). Both approximations yield acceptable results for sufficiently high drive levels and small bubble radii, as expected by neglecting terms of order $R_{0} / R_{\text {res }}$.

\section{Frequency dependence}

Equation (21) suggests that the growth-rate is proportional to the resonant radius and therefore scales as $1 / \omega$. This owes to the decrease of the bubble expansion ratio for increasing frequencies. To check this scaling and explore the validity range of our approximation, both numerical and analytical computations were repeated for frequencies of $50 \mathrm{kHz}$ and $100 \mathrm{kHz}$. The results are displayed in Fig. 5 and confirm this frequency dependence. Clearly, both approximations (19) and (21) (dashed line and dot-dashed line respectively) become worse, since the resonant radius decreases with frequency, so that the approximation $R_{0} / R_{\text {res }} \ll 1$ becomes unjustified. However, we emphasize that our full analytical formula (17) (thin solid line in Fig. 5) still yields an excellent approximation for the bubble growth-rate at these high frequencies. The noisy features of the numerical curves were found to origin from period-doubling bifurcations of the bubble dynamic [29-31]. In this case, rigorously, the time-averages used in the calculation of the growth-rate should be calculated on the smallest period of the system. We did not pursue further such a refinement, since it can be seen that even in this case, Eq. (17) still yields acceptable results.

\section{Estimation of the growth-time}

Finally, in view of practical applications, it is interesting to calculate the time necessary for an inertial bubble to grow between two ambient radii $R_{1}$ and $R_{2}$. To that aim, the approximate expression of the growth-rate (21) may be integrated analytically, which yields:

$$
\begin{aligned}
\Delta \tau\left(R_{1}, R_{2}\right)= & \frac{A}{9} \frac{p_{0}}{D R_{G} T C_{\infty}} \frac{1}{F_{2}(p) R_{\mathrm{res}}} \\
& \times\left[R_{2}^{3}\left(1+\frac{2 \sigma}{p_{0} R_{2}}\right)-R_{1}^{3}\left(1+\frac{2 \sigma}{p_{0} R_{1}}(2)\right]\right)
\end{aligned}
$$


Thus we get the surprisingly simple result that, apart from a surface tension correction, the growth-time of an inertial bubble between two sizes is directly proportional to the difference between the final and initial volume. To take a practical example, we calculate the time necessary for a bubble to grow from $1 \mu \mathrm{m}$ to $5 \mu \mathrm{m}$, the former being roughly of the order of the Blake threshold (and therefore of the RD threshold) and the latter corresponding to an approximate value of the shape instability threshold for drive levels of the order of 1.5 bar [15]. The results are presented in Fig. 6 which represents the growth-time in numbers of acoustic cycles for the three frequencies 26.5 $\mathrm{kHz}, 50 \mathrm{kHz}$ and $100 \mathrm{kHz}$.

Although the value of the fragmentation threshold is probably lower than $5 \mu \mathrm{m}$ for pressures higher than 1.5 bar, the present calculation yields an order of magnitude of the lifetime of a growing inertial bubble: for a $26.5 \mathrm{kHz}$ frequency and an acoustic pressure ranging between 1.2 and 5 bar, the growth-time ranges approximately from 20 to 150 acoustic cycles.

\section{DISCUSSION}

The above results bring several immediate consequences for multi-bubble fields in saturated water. First, since the rectified diffusion threshold radius merges with the Blake threshold radius (for acoustic pressures greater than 1.4 bar, see Fig. 1), the existence of growing smoothly oscillating bubbles is unlikely. This result casts some doubts on the role of rectified diffusion to initiate the cavitation cycle at high drive levels. Indeed, a small gas nucleus escaping from a solid crevice or a fragmentation debris falling below the common threshold would dissolve and never become inertial. Coalescence of such dissolving micro-bubbles driven under the Blake-RD threshold may constitute an alternative scenario of the construction and sustainment of the bubble field. This raises the issue of the dynamical competition between dissolution, which tends to kill the bubble population, and coalescence, which would restore bubbles above the

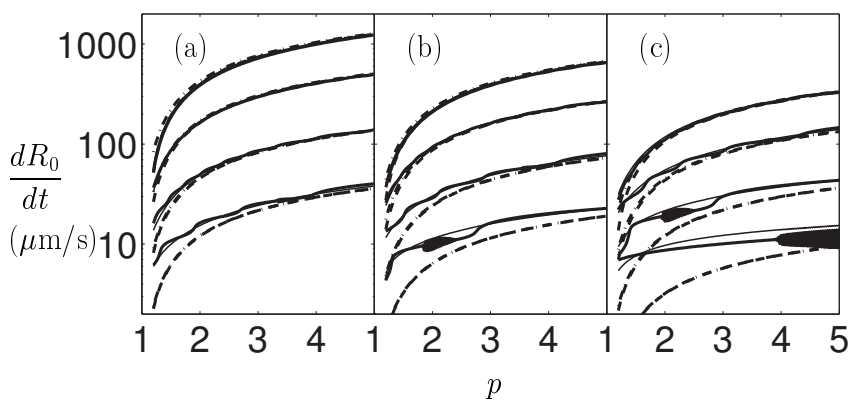

FIG. 5: Calculated value of the bubble growth-rate for different frequencies. (a) $f=26.5 \mathrm{kHz}$ (same as Fig. 4) (b) $f=$ $50 \mathrm{kHz}$ (c) $f=100 \mathrm{kHz}$. The line-style conventions are the same as Fig. 4. unique Blake-RD threshold.

In addition, the authors suggest that the above results shed a new light on the absence of SBSL emissions in multi-bubble fields. Rectification of argon in air bubbles have been proved essential to obtain SBSL [32, 33] and, as proposed by Matula and Crum [32], "multi-bubble sonoluminescence bubbles (MBSL) probably do not survive for more than a few cycles" so that argon rectification cannot occur in saturated multi-bubble fields. The point we would like to discuss is the reason for such a short lifetime of cavitation field bubbles. It is generally attributed, without clear theoretical ground, to early asymmetrical collapses caused by perturbations of neighboring bubbles [20], which would burst the bubble after a few collapses. Now, our calculation of the growth-time from the Blake threshold to the shape instability threshold yields an order of magnitude of tens or acoustic cycles for $26.5 \mathrm{kHz}$ acoustic fields. This is still much lower than the time necessary to rectify argon (several thousands of acoustic cycles following Ref. 32), and may also explain why SBSL cannot be observed in multi-bubble fields: we propose that the locus of shape instability of a cavitation field bubble could be the same as for a SBSL bubble (of course in the same conditions of amplitude and frequency), but that a cavitation field bubble, owing to the high gasconcentration, grows up to this locus too rapidly to rectify argon. Therefore, our interpretation differs from the conventional picture in that the shape instabilities destroying the cavitation bubbles need not be ascribed to the presence of other bubbles.

It might be argued that the case of a diffusivelyunstable SBSL bubble $[12,34]$ is apparented to cavitation field bubbles, as it also undergo cycles of growth phases followed by a fragmentation. However, unstable SBSL appears in degassed water and the growth-time in such conditions is much larger. Following figure 11 of Ref. [12], this time ranges between 2500 and 16000 cycles, depending on the degassing level, which is of the order of magnitude of the argon rectification time [32]. Therefore

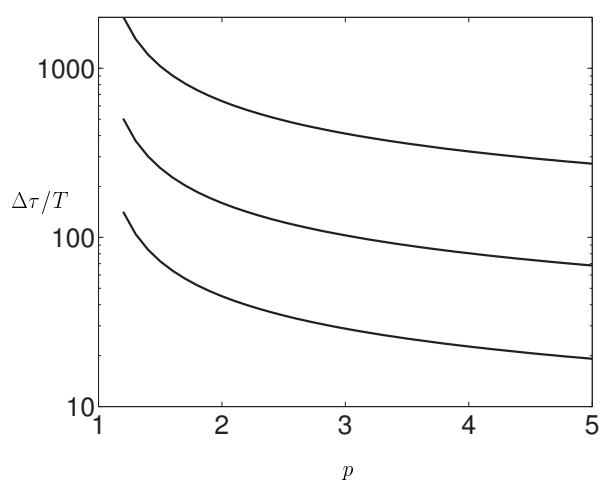

FIG. 6: Growth-time between $1 \mu \mathrm{m}$ and $5 \mu \mathrm{m}$ of an air-bubble in water expressed in numbers of acoustic cycles, calculated from Eq. (22). From bottom to top: $f=26.5 \mathrm{kHz}, f=50$ $\mathrm{kHz}$, and $f=100 \mathrm{kHz}$. 
argon rectification can still take place for unstable SBSL.

Besides, it has been mentioned that the background emission spectrum of MBSL bubbles shares some similarities with the SBSL spectrum, which would indicate the presence of a few SBSL-like bubbles in cavitation fields $[35,36]$. If, as suggested in this paper, such SBSL emission would be limited by the short lifetime of growing inertial air-bubbles, the use of higher frequencies would allow larger lifetimes, as attested by Eq. (22), and may allow a greater number of growing inertial bubbles to rectify argon before they hit the shape instability threshold. The author also share Matula and co-worker's suggestion [35] of MBSL experiments in sufficiently degassed liquids in order to enlarge the lifetime of the bubble.

It should be mentioned that the above results rely on the assumption of a unique pressure amplitude $p$, which is unrealistic in the case of multi-bubble fields, owing to standing wave effects. In fact, bubbles feel different drive levels as they travel in the acoustic field. A typical phenomenon related to this issue is the formation of filamentary streamer structures investigated by Lauterborn and co-workers [23, 37, 38]. If, as suggested by these authors, the bubbles constituting these filaments are inertial bubbles, which should be the case in view of the drive levels reported, the above theory predicts that these bubbles should be growing. Furthermore their growth might be further accelerated as they approach pressure antinodes under the influence of Bjerknes forces. From the above estimation, their lifetime would amount to several tens of cycles until they hit the shape stability threshold and burst. This raises the question of whether the bubbles constituting the filaments keep their spherical shape. It should be added that bubble collisions may also restrict the bubble lifetime. Indeed, from Luther et al.'s results [37], it can be conjectured that the time between collisions in a multi-bubble field configuration is of the order of hundreds of periods, so that this process may compete with the above-described one. In view of the time resolution of their experiment, the authors neither exclude that, on their way to the pressure antinode, bubbles may split off micro-bubbles. Numerical simulation may help to obtain a clear picture, and taking into account rectified diffusion in theoretical models of this phenomenon [23] may be a simpler matter by using the analytical formulae proposed in the present paper.

Besides, it is known that cavitation itself produces some level of degassing of the liquid, so that the quantity $C_{\infty}^{*}$ in the above expressions may decrease slowly during an experiment. Taking a generic value of $80 \%$ saturation at the end of the experiment, Eq. (22) shows that the growth-time would increase by a factor $5 / 4$, which does not change significantly the above conclusions.

Concerning the estimation of the surface instability threshold, one might argue that the choice of $5 \mu \mathrm{m}$ is rather rough, in view of the wide parameter range considered here. Following Lin et al. [15] (see their Fig. 2, dot-dashed curve), direct numerical simulation at 32.8 $\mathrm{kHz}$ yields this order of magnitude for drive levels rang- ing between 1.2 and 1.6 atm, in agreement with experimental results [19]. For higher pressure levels, the surface instability threshold radius is probably lower, so that our estimation of the bubble lifetime for $20 \mathrm{kHz}$ frequencies is probably overestimated, which reinforce our conclusion. Conversely, for higher frequencies, the authors are not aware of reported calculated or measured data, apart from the SBSL phase diagrams calculated by Prosperetti and Hao [14]. Their Fig. 19 suggest in indirect form that the surface instability threshold increases with frequency. This would mean that our estimation of the bubble lifetime for 50 and $100 \mathrm{kHz}$ may be underestimated, at least for moderate drive levels, and further supports our suggestion of possible higher SBSL-like emission for higher drive frequencies. Real calculations of the surface instability threshold at such frequencies would allow a firmer conclusion.

Finally, we would like to discuss the validity of the bubble model used throughout this paper. Concerning the isothermal assumption, we recall that the growthrate is mainly dominated by the expansion phase of the bubble. During this expansion, the liquid inertia predominates and the gas pressure is very low (and is indeed neglected in the analytical approach of the expansion phase in Ref. 4), so that it should be quasi-insensitive to thermal effects inside the bubble. There exists of course a frequency limit to this assumption since the linear and nonlinear resonance radii approach the Blake radius for increasing frequency. This may be put on more quantitative ground by repeating the above calculations by switching from isothermal to adiabatic behavior when the heat-diffusion time-scale becomes small [39].

Vapor exchange was also neglected in the present calculations. Recent calculations show that water may be trapped in the bubble during the collapse, mainly owing to finite diffusion between the center and the wall of the bubble, and also to non-equilibrium phase-change [26]. This water-trapping process may influence the bubble growth-rate, which in our approach is only due to gas accumulation, but its correct representation would also require the modeling of the chemical reactions occurring in the bubble and the diffusion of the reaction products out of the bubble.

\section{CONCLUSION}

The rectified diffusion threshold has been computed by numerical simulations of the Rayleigh-Plesset equation, for small bubbles $\left(R_{0}<15 \mu \mathrm{m}\right)$, low-frequency $(20 \mathrm{kHz}$ $<f<100 \mathrm{kHz}$ ) in a strong acoustic field ( 1 bar $<p<5$ bar). It appears that for near-saturated water, a growing bubble is necessarily oscillating inertially.

The growth-rate for such growing inertial bubbles has also been calculated, assuming that such bubbles may keep their integrity up to the shape instability threshold inferred from single-bubble theory. We also derived a fully explicit analytical formula for the growth-rate, 
showing excellent agreement with the exact numerical solution in a wide parameter range.

The merging of the two thresholds in near-saturation bubble-field experiments raises the question of how micro-bubbles driven under this single threshold can contribute to the construction of the cavitation field. Coalescence was proposed as a competing process to drive small nuclei above the common threshold.

An order of magnitude of the lifetime of growing inertial bubbles, from their inception at the Blake threshold, up to the instability threshold, has been calculated. It has been found to be much shorter than the time necessary to rectify argon. It was therefore suggested that the non occurrence of single-bubble sonoluminescence emission in multi-bubble fields may be due to this rapid growth-phase, without invoking any shape instability process caused by the presence of neighboring bubbles, as generally stated in the literature. The difference between single-bubble and multi-bubble has thus been interpreted in an alternative framework, based on the most immediate difference between the two situations: the gas saturation.

Finally, our analytical expression of the growth-rate is ready to use to account for the rectified diffusion in theoretical studies of cavitation clouds, based on population balance models [38, 40], or particle models [23]. It is interesting to note that the initial rather crude approximation of the bubble volume yields such a good approximation. This is due to the fact that the bubble dynamics only occurs as time-averages in the expression of the growth-rate. The method of approximation used in this paper is therefore promising for deriving analytical formulae of other quantities involving time-averages, such as primary and secondary Bjerknes forces for example. This may be the matter of a future work.

\section{Acknowledgments}

One of the authors (OL) would like to acknowledge Pr. Luis Gaete for hosting a pleasant stay in the University of Santiago de Chile. The authors would like to thank the anonymous referees for interesting comments.

\section{APPENDIX A: APPROXIMATE FORMULA FOR INTEGRAL $I$}

First, for simplicity, we will denote by $R_{\max }^{*}$ the expansion ratio:

$$
R_{\max }^{*}=\frac{R_{\max }}{R_{0}}
$$

In order to extract the main dependency of the integral on $R_{\max }^{*}$, we first make the following variable-change in integral (7):

$$
3 s=R_{\max }^{*} u,
$$

to obtain

$$
I=\frac{1}{3 R_{\max }^{*}} \int_{0}^{+\infty} \frac{d u}{G(u)}
$$

where

$$
G(u)=\frac{1}{2 \pi} \int_{0}^{2 \pi}\left(u+\left(\frac{R^{*}(x)}{R_{\max }^{*}}\right)^{3}\right)^{4 / 3} d x .
$$

We first focus on finding an approximation of the timeaverage $G(u)$. It only involves the quantity $R^{*}(x) / R_{\max }^{*}$ which by construction is bound in $[0,1]$. Figure $3 \mathrm{~b}$ suggests that we could approximate $R^{* 3}(x)$ by a parabola of maximum $R_{\max }^{*}{ }^{3}$ between $x_{m}-\Delta x$ and $x_{m}+\Delta x$, and by 0 anywhere else, so that we set:

$R^{* 3}(x) \simeq\left\{\begin{array}{l}R_{\max }^{*}{ }^{3}\left[1-\left(\frac{x-x_{m}}{\Delta x}\right)^{2}\right] \\ 0 \\ \text { if } x-x_{m} \in[-\Delta x, \Delta x] \\ \text { elsewhere }\end{array}\right.$

The time-interval $\Delta x$ should be chosen of the order of the characteristic time for the bubble expansion. We report further below the appropriate choice for this quantity.

We now calculate the time-average (A4) using this approximation. Cutting the acoustic cycle $[0,2 \pi]$ in three subintervals $\left[0, x_{m}-\Delta x\right],\left[x_{m}-\Delta x, x_{m}+\Delta x\right]$ and $\left[x_{m}+\Delta x, 2 \pi\right]$ we obtain:

$$
\begin{aligned}
G(u) \simeq & (1-\Delta x / \pi) u^{4 / 3} \\
+ & \frac{1}{2 \pi} \int_{x_{m}-\Delta x}^{x_{m}+\Delta x}\left[u+1-\left(\frac{x-x_{m}}{\Delta x}\right)^{2}\right]^{4 / 3}
\end{aligned}
$$

Then, by the variable change

$$
v=\left(\frac{x-x_{m}}{\Delta x}\right)^{2}
$$

the second integral in Eq. (A6) becomes:

$$
\frac{\Delta x}{\pi}(u+1)^{4 / 3} \int_{0}^{1}\left(1-\frac{v}{u+1}\right)^{4 / 3} \frac{d v}{\sqrt{v}},
$$

which may be recognized as the integral form of a hypergeometric function:

$$
{ }_{1} F_{2}(a, b ; c ; z)=\frac{\Gamma(c)}{\Gamma(b) \Gamma(b-c)} \int_{0}^{1} \frac{v^{b-1}(1-v)^{c-b-1}}{(1-v z)^{a}} d v .
$$


We finally obtain, as an approximation of the timeaverage quantity (A4):

$$
\begin{aligned}
G(u) \simeq & \left(1-\frac{\Delta x}{\pi}\right) u^{4 / 3}+\frac{\Delta x}{\pi}(u+1)^{4 / 3} \\
& \times{ }_{1} F_{2}\left(-\frac{4}{3}, \frac{1}{2} ; \frac{3}{2} ; \frac{1}{u+1}\right),
\end{aligned}
$$

and integral $I$ may be written as:

$$
I \simeq \frac{1}{3 R_{\max }^{*}} \int_{0}^{+\infty} \frac{d u}{\left(1-\frac{\Delta x}{\pi}\right) u^{4 / 3}+\frac{\Delta x}{\pi}(u+1)^{4 / 3}{ }_{1} F_{2}\left(-\frac{4}{3}, \frac{1}{2} ; \frac{3}{2} ; \frac{1}{u+1}\right)} .
$$

Any further progress may look difficult, but it's worth recalling that $\Delta x$ is of the order of magnitude of the expansion time of the bubble, so that it belongs anyway to the interval $[0, \pi]$ (except for very high pressure drives). We thus calculated the integral (A11) for values of the parameter $\Delta x$ in this interval. The numerical method used is presented in appendix B . Fitting the result by a power-law we finally obtain

$$
I \simeq \frac{1}{3 R_{\max }^{*}} \frac{A}{\Delta x^{e}},
$$

with $e=0.37$ and $A=0.90$. We emphasize that this fit is not linked to any specific choice of parameters $R_{0}$ or $p$ and that the approximate expression (A12) is accurate provided the expansion phase dominates the bubble dynamics, that is to say for any inertial bubble.

The last step is to choose the interval $\Delta x$ so that Eq. (A12) yields a good approximation of $I$. It should be said first that the dependence of the integral $I$ on the bubble dynamics is mainly caught by the scaling law $I=$ $O\left(R_{\max }^{*}{ }^{-1}\right)$, and that the dependence on $\Delta x$ is weaker, as attested by Eq. (A12). As mentioned above, the length of this interval is of the order of the expansion phase of the bubble, which was studied analytically in Ref. 4 (see also appendix $\mathrm{C}$ ). We choose:

$$
\Delta x=x_{m}-\alpha x_{+},
$$

where $x_{m}$ is the time of maximum expansion of the bubble, $x_{+}=\arccos (1 / p)$ is the zero-crossing of the acoustic pressure (see Fig. 3c), and $\alpha$ is a correcting factor to take into account the non parabolic shape of $R^{* 3}(x)$. The final approximation of $I$ was found weakly sensitive to the precise value of $\alpha$. A value of $\alpha=0.6$ yielded an excellent uniform agreement over the parameter range studied. Combining Eqs. (A12)-(A13) yields the final result Eq. (14).

Figure 7 shows the evolution of the integral $I$ vs. $R_{0}$, calculated directly from the RP equation (thick solid line), and from the approximate expression (14), for which the parameters $R_{\max }^{*}$ and $x_{m}$ were extracted from the numerical solution of the RP equation (thin solid line). It is seen that the two curves are in excellent agreement in most of the parameter range above the Blake threshold (represented by the dashed vertical lines for each $p$ value in Fig. 4). Figure 8 displays the same quantities with $p$ in abscissa for fixed $R_{0}$.

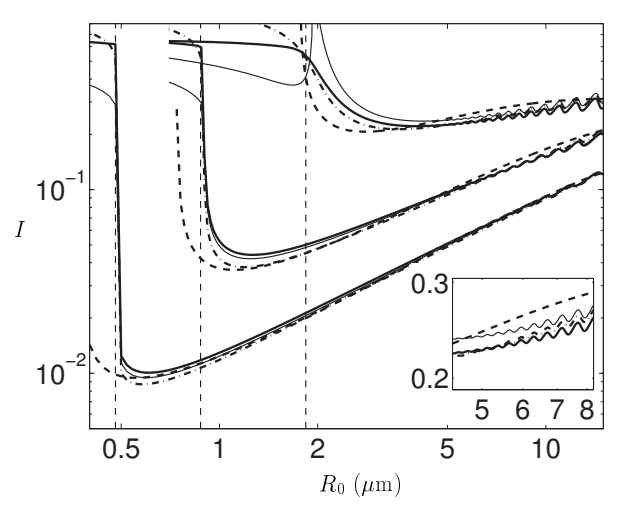

FIG. 7: Comparison of the exact value of $I$ calculated numerically (thick solid line) with approximate expressions of $I$ for $p=1.2$ (upper set of curves), $p=1.5$ (middle), and $p=2$ (lower). Thin solid line: Eq. (14) with $R_{\max }^{*}$ and $x_{m}$ calculated from the RP equation; dot-dashed line: Eq. (14) with $x_{m}$ calculated from Eq. (15b); dashed line: Eq. (14) with $R_{\max }^{*}$ and $x_{m}$ calculated from Eqs. (15a) and (15b). The three vertical thin dashed curves represent the Blake radii corresponding to $p=2,1.5$, and 1.2 (from left to right).

Figures 7 and 8 also show the values of $I$ calculated from: i) (14)-(15b), the expansion ratio $R_{\max }^{*}$ being deduced from the numerical RP solution (dot-dashed line); ii) from the fully analytical set of equations (14)-(15a,b) (dotted line). It appears that the latter choice yields an excellent, fully analytic approximation for $I$, except below the Blake threshold, where the approximation (15a) of the expansion ratio becomes less accurate. 


\section{APPENDIX B: NUMERICAL METHOD}

The numerical method was designed to calculate both the exact integral $I$ given by Eq. (7) and the approximate one (A11). In both cases, we are to compute integrals of the form:

$$
J=\int_{0}^{+\infty} \frac{d u}{H(u)},
$$

where $H(u)$ is either given by Eq. (A4) or by Eq. (A10). The integral (B1) is first transformed to an integral from -1 to 1 by the variable change:

$$
x=2 e^{-u}-1,
$$

to obtain

$$
J=\int_{-1}^{1} \frac{d x}{(1+x) H\left(-\log \left(\frac{1+x}{2}\right)\right)} .
$$

Then the integral was recast to fit to a Gauss-Jacobi quadrature formula:

$$
\int_{-1}^{1}(1-x)^{\alpha}(1+x)^{\beta} g(x) d x=\sum_{n=1}^{N} w_{n} g\left(x_{n}\right),
$$

where $w_{n}$ are the weights and $x_{n}$ the evaluation points, and $g$ reads

$$
g(x)=\frac{1}{(1-x)^{\alpha}(1+x)^{\beta+1} H\left(-\log \left(\frac{1+x}{2}\right)\right)} .
$$

The choice of $\alpha$ and $\beta$ is free (provided they are greater than -1) but good convergence of Gauss formulae strongly depends on the polynomial character of function $g$. We

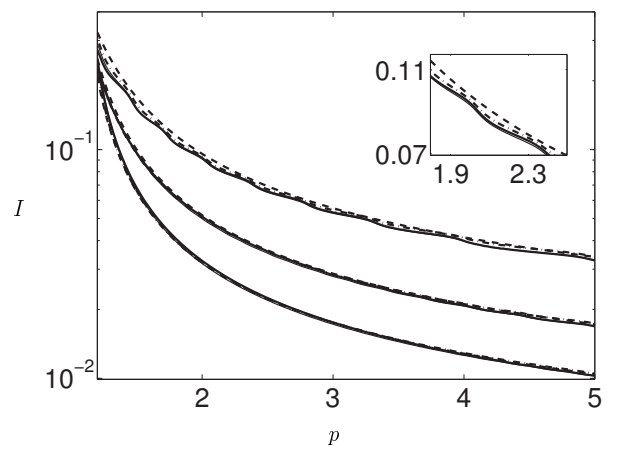

FIG. 8: Same as Fig. 7 with driving pressure in abscissa for fixed $R_{0}$. From bottom to top: $R_{0}=3,5$, and $10 \mu \mathrm{m}$. adjust the $\alpha$ and $\beta$ exponents by testing the polynomial character of $g$ for $H$ given by (A10). Figure 9 shows the evolution of $g$ with $\alpha=0$ and $\beta=-0.8$ for $\Delta x=$ $0.1,0.3$, and 0.5 . It is seen that with this values, the function is very smooth, which ensures the quality of the Gauss-Jacobi formula.

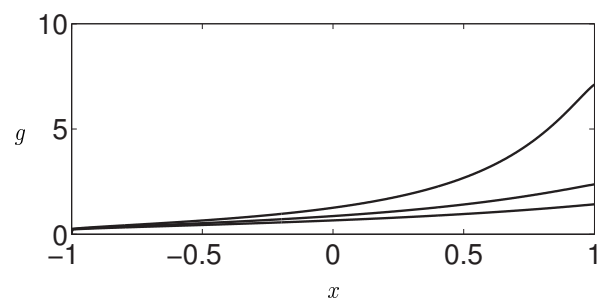

FIG. 9: Evolution of function $g$ in the interval $[-1,1]$ for $\Delta x=0.2 \pi, 0.6 \pi$, and $\pi$ (from top to bottom).

Equation (B4) was therefore used with such values of $\alpha$ and $\beta$ for the evaluation of both integrals (A3) and (A11). A convergence study showed that eleven terms in the sum (B4) were sufficient in all cases.

\section{APPENDIX C: APPROXIMATE FORMULAE FOR $x_{m}$ AND $R_{\max }^{*}$}

Hilgenfeldt et al. [4] showed that during the expansion phase, the gas-pressure, radiation and viscous term could be neglected in the RP equation, so that it could be reduced to the simpler form:

$$
R \ddot{R}+\frac{3}{2} \dot{R}^{2}=\frac{p_{0}}{\rho}\left[p \cos \omega t-\left(1+\frac{\alpha_{S}}{K(p)}\right)\right],
$$

where the last term accounts for surface tension effects, with $\alpha_{S}=2 \sigma / p_{0} / R_{0}$. They observed numerically that $R \ddot{R} \ll \dot{R}$ for $\omega t \in\left[-x_{+}, x_{+}\right]$, while $R \ddot{R} \gg \dot{R}$ for $\omega t \in$ $\left[x_{+}, x_{m}\right]$, where $x_{+}=\arccos (1 / p)$ and $x_{m}$ is the time of maximum expansion of the bubble (see Fig. 3). Further observing that $R \ddot{R}+\dot{R}^{2}=d^{2}\left(R^{2} / 2\right) / d t^{2}$, the simplified ODE (C1) could be integrated in these intervals. By matching the boundary conditions at $-x_{+}, x_{+}$, and $x_{m}$, they obtained the following expressions for the maximum radius and the time of maximum expansion [41]:

$$
\begin{aligned}
R_{\max }^{*}= & f\left(p, x_{m}\right)+g\left(p, x_{m}\right) \\
& \times\left[1-\frac{4}{9 \sqrt{3}} \frac{\alpha_{S}}{p-1}\right] \frac{R_{\mathrm{res}}^{2}}{R_{0}^{2}}, \\
p \sin x_{m}- & x_{m}+\frac{1}{3}\left(p \sin x_{+}-x_{+}\right) \\
& +3 \zeta^{2}\left(\frac{R_{0}}{R_{\mathrm{res}}}\right)^{2}=0
\end{aligned}
$$

where $\zeta=1.6$ is an adjustable parameter, $R_{\text {res }}=$ $\left(3 p_{0} / \rho \omega^{2}\right)^{1 / 2}$ is the isothermal resonant radius and functions $f$ and $g$ read: 


$$
\begin{aligned}
f\left(p, x_{m}\right)= & \zeta^{2}\left[1+2\left(x_{m}+x_{+}\right)\right] \\
g\left(p, x_{m}\right)= & \frac{2}{3}\left[1-p \cos x_{m}-\frac{1}{2}\left(x_{m}^{2}-x_{+}^{2}\right)\right. \\
& \left.+\frac{1}{3}\left(p \sin x_{+}-x_{+}\right)\left(x_{m}+3 x_{+}\right)\right]
\end{aligned}
$$

Equation (C2b) in implicit in $x_{m}$ and Hilgenfeldt et al. [4] deduced several particular cases for either small or large values of $p$. Here, we seek an explicit approximation uniformly valid at least in the interval $p \in[1,5]$. We follow the method of Ref. 4 by expanding $x_{m}$ around $\pi / 2$ but here, we use an expansion up to second order, and obtain for $x_{m}$ :

$$
x_{m}=\frac{\pi}{2}+\frac{\sqrt{1+p\left(Q(p)-\pi+6 \zeta^{2} R_{0}^{2} / R_{\mathrm{res}}^{2}\right)}-1}{p},
$$

where

$$
Q(p)=2 p+\frac{2}{3}\left(p \sin x_{+}-x_{+}\right) .
$$

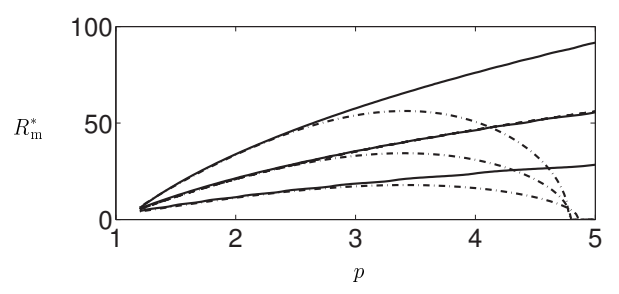

[1] F. G. Blake, Harvard Univ. Acoust. Res. Lab. Tech. Mem. (1949).

[2] E. A. Neppiras, Phys. Rep. 61, 159 (1980).

[3] I. Akhatov, N. Gumerov, C. Ohl, U. Parlitz, and W. Lauterborn, Phys. Rev. Lett. 78, 227 (1997).

[4] S. Hilgenfeldt, M. P. Brenner, S. Grossman, and D. Lohse, J. Fluid Mech. 365, 171 (1998).

[5] D. Y. Hsieh and M. S. Plesset, J. Acoust. Soc. Am. 33, 206 (1961).

[6] A. Eller and H. G. Flynn, J. Acoust. Soc. Am. 37, 493 (1965).

[7] L. A. Crum and G. M. Hansen, J. Acoust. Soc. Am. 72, 1586 (1982)

[8] M. M. Fyrillas and A. J. Szeri, J. Fluid Mech. 277, 381 (1994).

[9] R. E. Apfel, J. Acoust. Soc. Am. 69, 1624 (1981).

[10] T. J. Leighton, The acoustic bubble (Academic Press, 1994).

[11] D. F. Gaitan, L. A. Crum, C. C. Church, and R. A. Roy, J. Acoust. Soc. Am. 91, 3166 (1992).

[12] S. Hilgenfeldt, D. Lohse, and M. P. Brenner, Phys. Fluids 8, 2808 (1996).
FIG. 10: Expansion ratio $R_{\max }^{*}$ calculated from numerical solution of the RP equation (solid line), and from approximation (C2a)-(C6) (dashed line) for $R_{0}=3 \mu \mathrm{m}, 5 \mu \mathrm{m}$, and $10 \mu \mathrm{m}$ (from top to bottom). The dot dashed curves are calculated from (C2a) with $x_{m}=p$ (see Ref. 4).

In order to eliminate the dependence of $x_{m}$ in $R_{0}$, we further neglect the $R_{0}^{2} / R_{\text {res }}^{2}$ term in the square root of Eq. (C4), since for low frequencies bubbles near the Blake threshold are much smaller than the resonant radius and we finally get:

$$
x_{m}=h(p)=\frac{\pi}{2}+\frac{\sqrt{1+p(Q(p)-\pi)}-1}{p} .
$$

Replacing $x_{m}$ by Eq. (C6) in Eqs. (C3a,b), equation (C2a) becomes an analytical expression for $R_{\max }^{*}$, which is used throughout this paper. The result (dashed line) is compared to numerical exact solution (solid line) in Fig. 10: the two curves are indistinguishable. Also shown is the approximation obtained by taking $x_{m}=p$ in Eq. (C2a) (dot-dashed line) [4]. It is seen that expanding $x_{m}$ around $\pi / 2$ up to the second order as we did here, improves drastically the approximation for larger $p$.

[13] C. C. Wu and P. H. Roberts, Phys. Lett. A 250, 131 (1998).

[14] A. Prosperetti and Y. Hao, Phil. Trans. R. Soc. Lond. A 357, 203 (1999).

[15] H. Lin, B. D. Storey, and A. J. Szeri, Phys. Fluids 14, 2925 (2002)

[16] M. S. Plesset, J. Appl. Phys. 25, 96 (1954).

[17] A. Prosperetti, Q. Appl. Math. 34, 339 (1977).

[18] R. G. Holt and D. F. Gaitan, Phys. Rev. Lett. 77, 3791 (1996).

[19] J. A. Ketterling and R. E. Apfel, Phys. Rev. E 61, 3832 (2000).

[20] L. A. Crum, J. Acoust. Soc. Am. 95, 559 (1994).

[21] R. Mettin, I. Akhatov, U. Parlitz, C. D. Ohl, and W. Lauterborn, Phys. Rev. E 56, 2924 (1997).

[22] F. Burdin, N. A. Tsochatzidis, P. Guiraud, A. M. Wilhelm, and H. Delmas, Ultrason. Sonochem. 6, 43 (1999).

[23] U. Parlitz, R. Mettin, S. Luther, I. Akhatov, M. Voss, and W. Lauterborn, Phil. Trans. R. Soc. Lond. A 357, 313 (1999).

[24] H. Lin, B. D. Storey, and A. J. Szeri, J. Fluid Mech. 452, 145 (2002). 
[25] A. Prosperetti, J. Fluid. Mech. 222, 587 (1991).

[26] B. D. Storey and A. Szeri, Proc. R. Soc. Lond. A 456 1685 (2000).

[27] R. Löfstedt, B. P. Barber, and S. J. Putterman, Phys. Fluids A5, 2911 (1993).

[28] C. C. Church, J. Acoust. Soc. Am. 83, 2210 (1988).

[29] W. Lauterborn and E. Cramer, Phys. Rev. Lett. 47, 1445 (1981).

[30] V. I. Ilyichev, V. L. Koretz, and N. P. Melnikov, Ultrasonics 27, 357 (1989).

[31] U. Parlitz, V. English, C. Scheffczyk, and W. Lauterborn, J. Acoust. Soc. Am. 88, 1061 (1990)

[32] T. J. Matula and L. A. Crum, Phys. Rev. Lett. 80, 865 (1998).

[33] B. D. Storey and A. J. Szeri, Phys. Rev. Lett. 88, 074301 (2002).

[34] B. P. Barber, K. R. Weninger, S. J. Putterman, and
R. Löfstedt, Phys. Rev. Lett. 74, 5276 (1995).

[35] T. J. Matula, R. A. Roy, P. D. Mourad, W. B. McNamara, and K. S. Suslick, Phys. Rev. Lett. 75, 2602 (1995).

[36] T. J. Matula and R. A. Roy, Ultrason. Sonochem. 4, 61 (1997).

[37] S. Luther, R. Mettin, P. Koch, and W. Lauterborn, Ultrason. Sonochem. 8, 159 (2001).

[38] I. Akhatov, U. Parlitz, and W. Lauterborn, Phys. Rev. E 54, 4990 (1996)

[39] B. D. Storey and A. Szeri, Proc. R. Soc. Lond. A 457, 1685 (2001)

[40] Y. A. Kobelev and L. A. Ostrovskii, J. Acoust. Soc. Am. 85, 621 (1989).

[41] We take this opportunity to report what we think is a mistake in Ref. 4: the $+\frac{2}{3} \frac{\alpha_{S}}{K(p)}$ appearing in the bracket of their Eq. (4.34) should be replaced by $-\frac{\alpha_{S}}{K(p)}$. 